\title{
Recent insights into the tick microbiome gained through next-generation sequencing
}

Telleasha L. Greay*, Alexander W. Gofton, Andrea Paparini, Una M. Ryan, Charlotte L. Oskam and Peter J. Irwin

\begin{abstract}
The tick microbiome comprises communities of microorganisms, including viruses, bacteria and eukaryotes, and is being elucidated through modern molecular techniques. The advent of next-generation sequencing (NGS) technologies has enabled the genes and genomes within these microbial communities to be explored in a rapid and cost-effective manner. The advantages of using NGS to investigate microbiomes surpass the traditional nonmolecular methods that are limited in their sensitivity, and conventional molecular approaches that are limited in their scalability. In recent years the number of studies using NGS to investigate the microbial diversity and composition of ticks has expanded. Here, we provide a review of NGS strategies for tick microbiome studies and discuss the recent findings from tick NGS investigations, including the bacterial diversity and composition, influential factors, and implications of the tick microbiome.
\end{abstract}

Keywords: Ticks, Microbiome, Next-generation sequencing, Bacteria, Protozoa, Viruses

\section{Background}

A microbiome, or microbiota, can be defined as a community of commensal, symbiotic, and pathogenic microorganisms [1-3]. The tick microbiome consists of communities of viruses, bacteria and eukaryotes [4], and tick-borne pathogens (TBPs) of humans and animals are among the most important microorganisms that have been characterised within these arthropod vectors. Such pathogens of medical and veterinary importance include Crimean-Congo haemorrhagic fever virus (CCHFV), Kyasanur Forest disease virus (KFDV), and species of Anaplasma, Borrelia, Coxiella, Ehrlichia, Francisella, Rickettsia, Babesia and Theileria [5-7]. Importantly, however, the most dominant microorganisms that reside in ticks are obligate endosymbionts [8-10].

As microbiomes are typically composed of hundreds of microbial species [11, 12], laboratory methods that are capable of identifying multiple species simultaneously in a single assay offer great advantages. The development of next-generation sequencing (NGS) technologies in the last decade has enabled the genes and genomes of single cells

\footnotetext{
* Correspondence: t.greay@murdoch.edu.au

Vector and Waterborne Pathogens Research Group, School of Veterinary and Life Sciences, Murdoch University, Perth, WA, Australia
}

and communities of microorganisms to be explored in a massively parallel, rapid, and cost-effective manner. Following the first NGS study of a tick microbiome in 2011 [13], the number of studies that have used NGS to investigate tick microbiomes has expanded (Table 1). Despite the growing number of studies, the microbiomes of most tick species remain unexplored, and further research into the functional role of these microorganisms in ticks at an individual and community level is needed.

This review compares the advantages and disadvantages associated with NGS platform selection and sequencing methods (amplicon and shotgun sequencing). The use of NGS data to characterise the tick microbiome through measures of diversity and composition is discussed and recent discoveries of bacteria that are pathogenic, symbiotic, novel and associated with tick organs are highlighted. We also consider aspects of the tick microbiome that are largely unexplored and factors that influence the structure and organisation of the microbiome.

\section{Microbiome next-generation sequencing strategies}

Two types of NGS can be applied to investigate microbiomes, amplicon sequencing and shotgun sequencing 


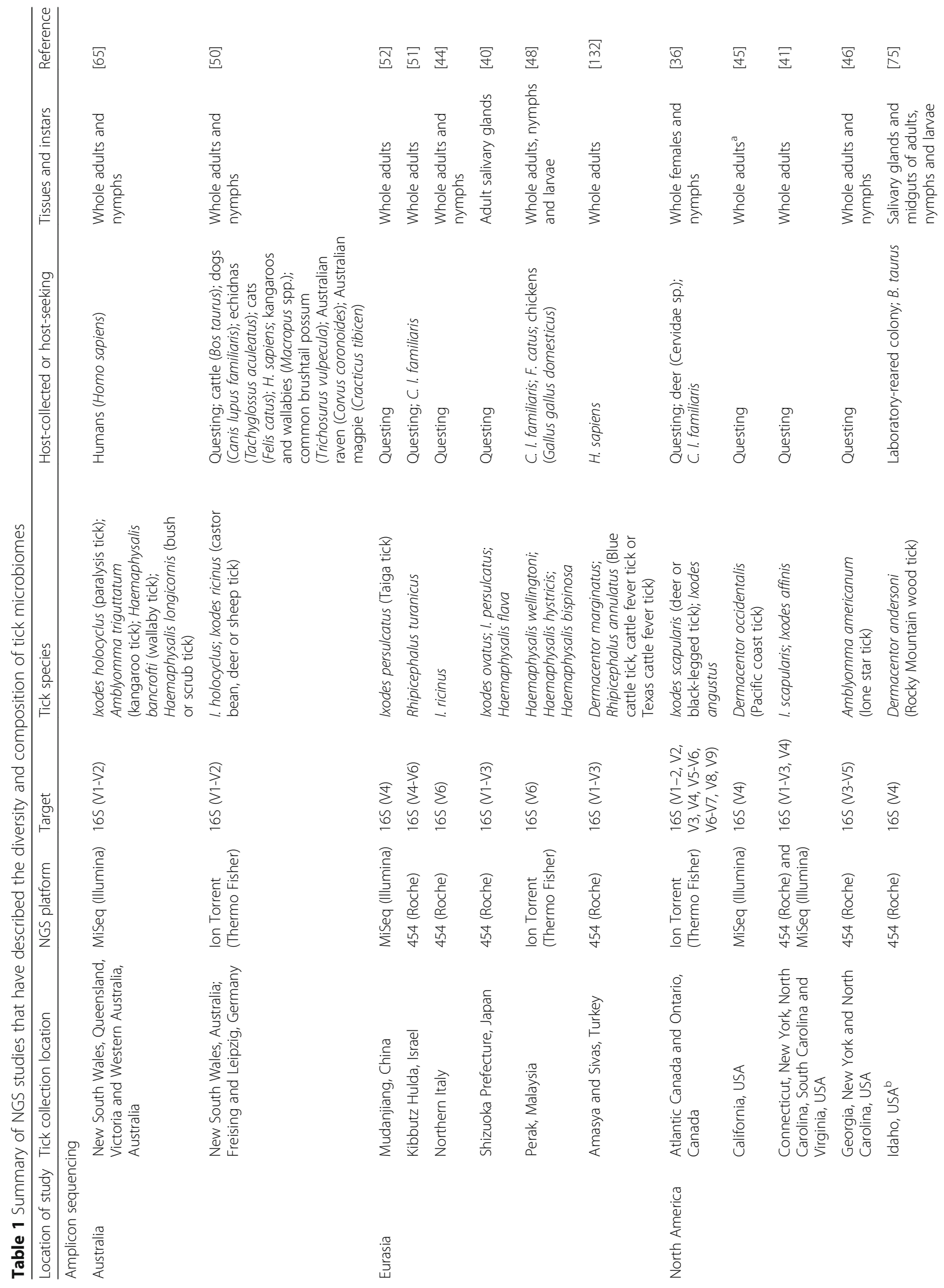




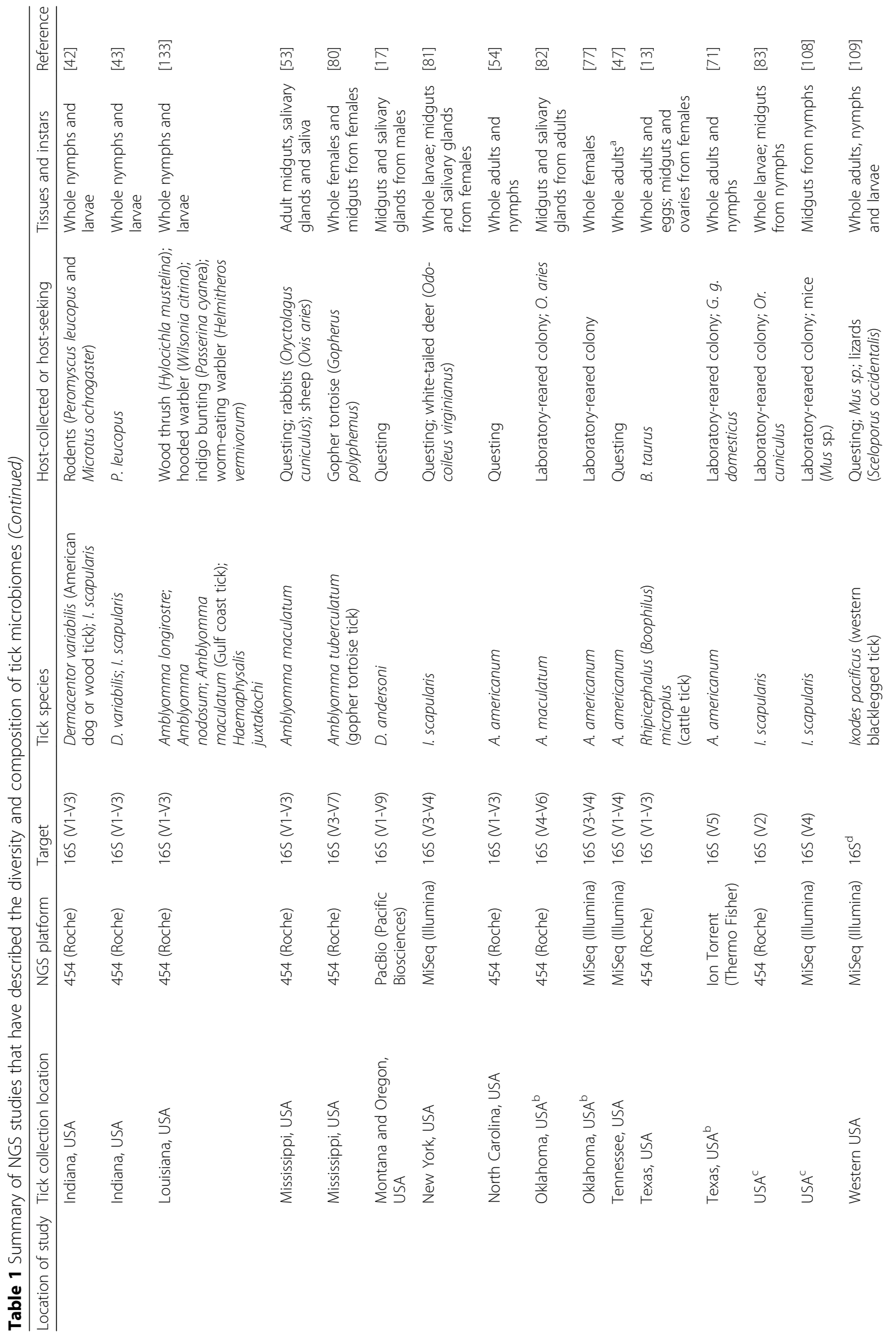




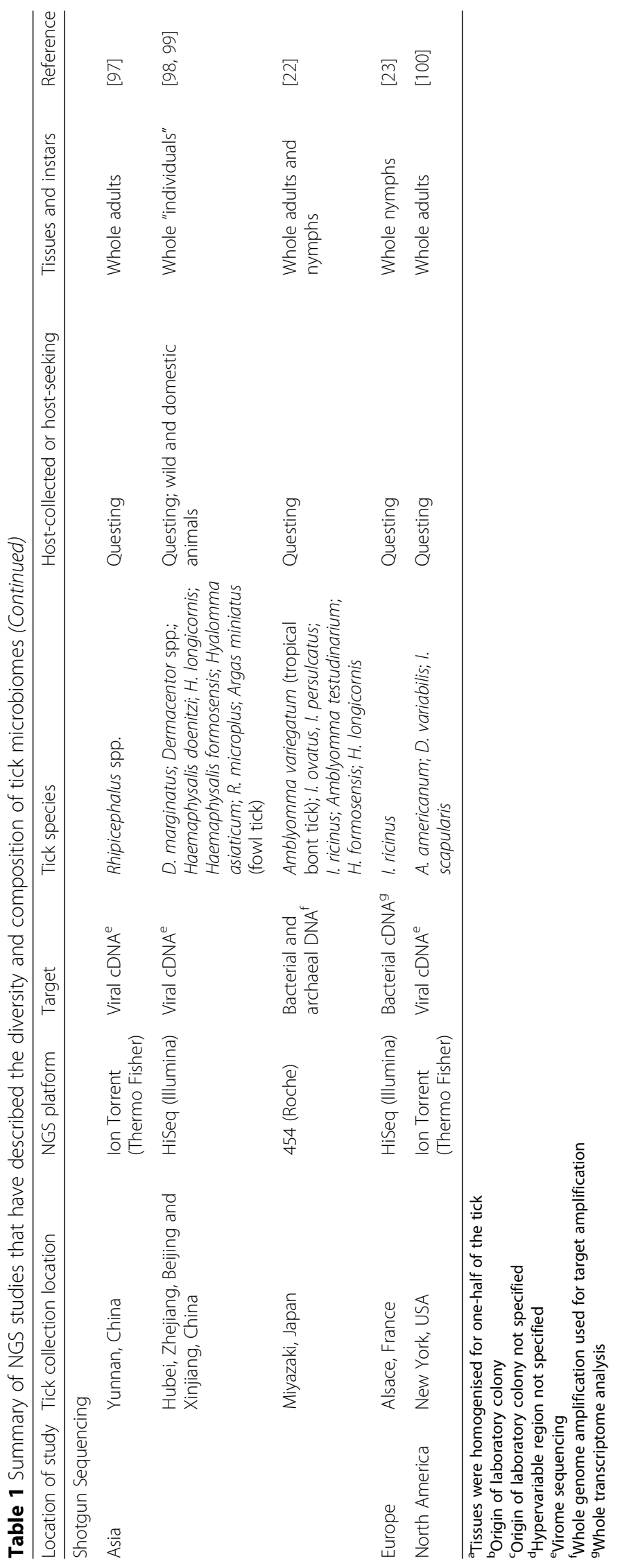


(which includes metagenomics and transcriptomics). There are nine hypervariable regions (V1-V9) of the bacterial $16 \mathrm{~S}$ ribosomal RNA gene (16S) that can be targeted to identify bacterial taxa in $16 \mathrm{~S}$ amplicon NGS studies, and regions V1-V4 have been most commonly sequenced in ticks (Table 1). 16S amplicon sequencing in ticks has been performed with 454 (Roche) pyrosequencing, Ion Torrent (Thermo Fisher) sequencing by semiconductor ion detection, and MiSeq (Illumina) platforms that use fluorescent dye detection sequencing methods (Table 1). Most of the published bacterial microbiome studies on ticks have used 454 platforms, such as the 454 GS Junior + and 454 GS FLX Titanium $\mathrm{XL}+$, which have the advantage of longer read lengths (up to $1 \mathrm{kbp}$ ) compared to the Ion Torrent and MiSeq platforms [14], however, these sequencers have been discontinued. The longest read lengths achieved by Illumina platforms are up to $600 \mathrm{bp}$ (single-end reads; 300 bp paired-end reads) on the MiSeq (with v3 chemistry) [15], and read lengths of up to $400 \mathrm{bp}$ (single-end reads) can be sequenced on the Ion Torrent platforms Ion PGM and Ion S5 [16]. Greater 16S read lengths can improve taxonomic resolution of the sequences, and although almost full length $16 \mathrm{~S}$ sequencing has been achieved on other platforms such as PacBio (Pacific Biosciences) [17], the cost of sequencing long amplicons is considerably more, and longer reads are provided at the expense of output read number [18], which is an equally important consideration for microbiome studies. The output read number is important for capturing adequate microbiome diversity, and the read numbers provided by the MiSeq are much greater than the Ion Torrent platform with comparable maximum read lengths; the MiSeq is capable of up to 50 million paired-end reads [15], whereas the Ion S5 530 platform can achieve up to 20 million single-end reads [16]. For shotgun sequencing, the HiSeq X (Illumina) sequences 150 bp pairedend reads and has the highest read output (2.6-3 billion reads) and lowest cost of sequencing (US\$7 per Gbp) compared to other shotgun sequencing platforms $[18$, 19]. A large number of output reads is important in shotgun sequencing for better metagenome (or transcriptome) coverage. PacBio [20] and MinION (Oxford Nanopore) [21] are single-molecule long-read sequencers, with read lengths of $\sim 20 \mathrm{kbp}$ and $200 \mathrm{kbp}$, respectively, that can be used for metagenomics. Although the output number of reads are low on these platforms (< 350,000 sequences), superior genome coverage is afforded by greater read lengths; however, these platforms are considerably more expensive than shortread sequencing technologies [18]. At present, only short-read sequencers have been used for shotgun sequencing of tick metagenomes, transcriptomes and viromes (Table 1).
Shotgun sequencing offers some advantages over amplicon sequencing such as the assessment of whole or partial genomes, transcriptomes, or viromes of the microbiome [22], and the sequencing of genes that are transcribed in microbes implies that they are actively replicating within the tick [23]. Furthermore, library preparation kits that are PCR-free can be used for shotgun sequencing library preparation [24], which eliminates the issues of PCR bias that amplicon sequencing is subjected to. However, at the current time, shotgun sequencing is considerably more expensive than amplicon sequencing [18], which may explain why the latter has been more widely used. Also, the nucleic acid extraction procedures for shotgun sequencing are more complex and require additional purification or enrichment steps compared to genomic DNA (gDNA) extraction methods for $16 \mathrm{~S}$ amplicon sequencing. Purification or enrichment of microbial DNA needs to be performed for shotgun sequencing of viral and microbial metagenomes, as well as metatranscriptomes, to increase the genome, virome, or transcriptome coverage by reducing eukaryotic DNA from the tick and from the tick's host [25-28]. Shotgun sequencing also results in significantly larger datasets than amplicon sequencing, which requires much more powerful computational tools for data storage and bioinformatic analyses. The approaches and software packages for NGS bioinformatics have been reviewed elsewhere [19, 29]. A schematic of the workflow for tick microbiome NGS studies is outlined in Fig. 1.

\section{Bacterial diversity}

The diversity of the microbiome can be assessed through measures of alpha and beta diversity; alpha diversity measures the number of species in a sample and their proportion (species richness), while beta diversity measures the dissimilarity between samples (genetic relatedness) [30-32]. Diversity metrics depend on the taxonomic resolution of sequences and sequencing depth [33]. 16S NGS of one to three hypervariable regions results in read lengths of $\sim 200-500 \mathrm{bp}$, which is a sufficient length for the taxonomic resolution of many, but not all, bacterial species. The nine hypervariable regions of $16 \mathrm{~S}$ that enable bacterial taxa to be identified exhibit varying degrees of sequence diversity, and unfortunately, no single hypervariable region can be used to distinguish between all bacterial species $[34,35]$. Although regions V1-V4 have been most commonly targeted in tick microbiome studies, a recent study that compared the bacterial diversity obtained from sequencing regions V1-V9 on the Ion Torrent found that regions V2, V3, V4, V6-V7, V8 and V9 gave the most comprehensive estimates of bacterial families, and the V4 region resulted in the highest estimated diversity [36]. Moreover, within some bacterial genera, the 


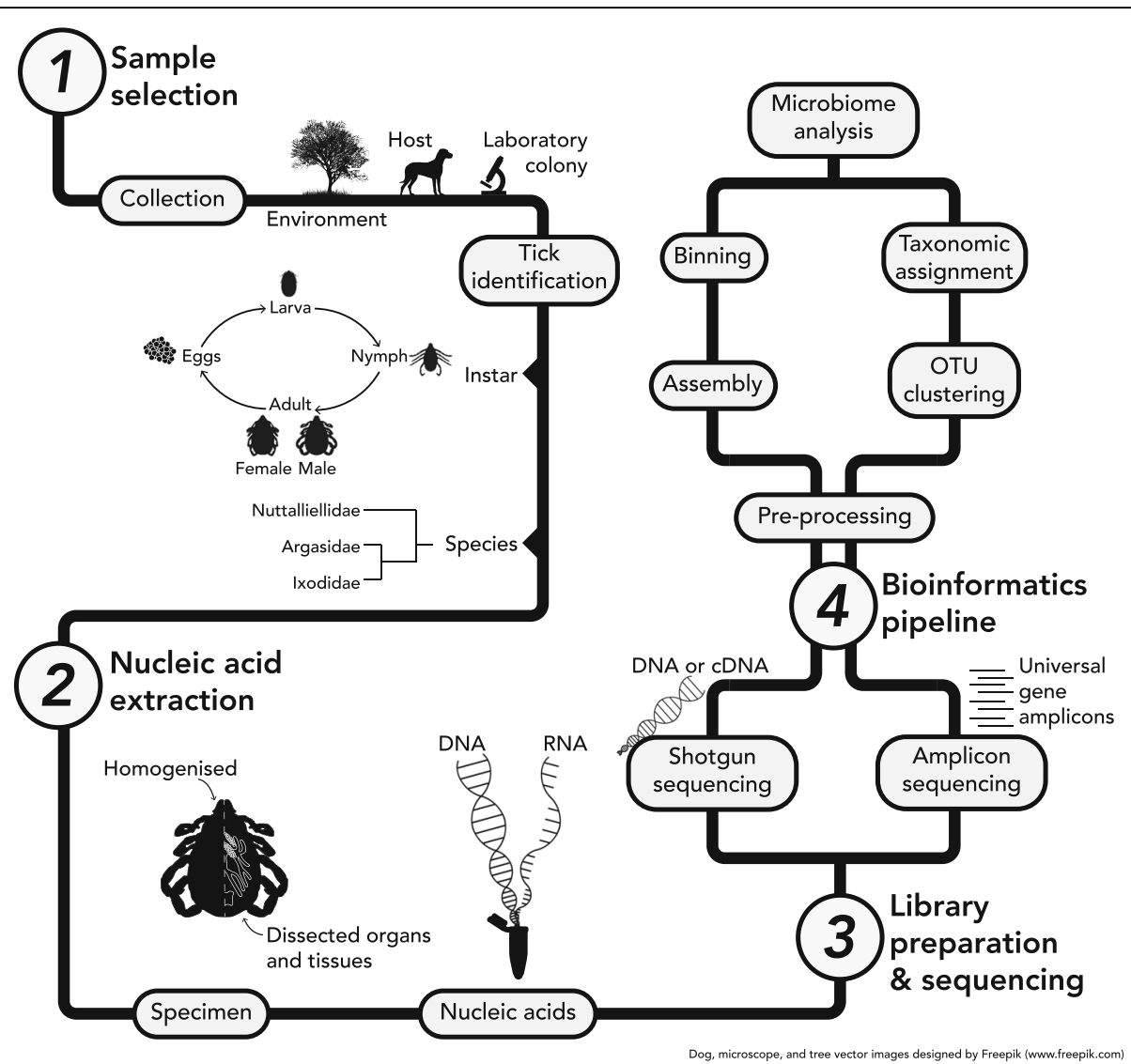

Fig. 1 Schematic of the NGS workflow for studying the tick microbiome

hypervariable regions of $16 \mathrm{~S}$ are highly conserved between species, which limits species-level identification, e.g. the genus Rickettsia, especially the spotted fever group rickettsiae (SFGR) [37]. Additionally, the choice of similarity cut-offs and clustering algorithms used to pick operational taxonomic units (OTUs) $[38,39]$ and sequencing error rates [33] can also affect taxonomic resolution.

Obtaining a sufficient number of reads per sample (referred to as depth, or coverage, in amplicon NGS studies) is required for adequate measurements of diversity [33]. Alpha diversity rarefaction plots, which compare the number of OTUs obtained to sequencing depth, should be assessed to determine whether adequate sequencing depth was achieved for each sample, and these plots can be produced using Quantitative Insights Into Microbial Ecology (QIIME) software. A plateau in the plot suggests that adequate depth was achieved, whereas increasing trends suggest that further sequencing depth is required. Since the microbial diversity can vary in ticks based on factors such as species [40-43] and geographical location [41, 44-47], the required sequencing depth differs between studies. For example, the alpha diversity rarefaction plots from the study by Rynkiewicz et al. [43] plateau at $\sim 20,000$ reads for Ixodes scapularis (deer or black-legged tick) and Dermacentor variabilis (American dog or wood tick), whereas the alpha diversity rarefaction plots for most samples in the study by Khoo et al. [48] have an increasing trend up to 40,000 reads for Haemaphysalis spp. However, underestimation of diversity can result when highly abundant sequences are overrepresented, regardless of sequencing depth. In such situations, PCR may not be sufficiently sensitive to amplify less abundant species. Blocking primers, which are primers that are modified with a DNA oligonucleotide or clamping probe that do not prime amplification, can be designed to reduce the amount of amplification of abundant template DNA during PCR [49]. This approach in Ixodes spp. has been shown to result in a significant increase in the total detectable bacterial diversity [50].

\section{Bacterial composition}

The bacterial composition, or relative abundance of the taxa identified, refers to the proportion of reads for each taxon relative to the total number of reads for all taxa and can be assessed for each individual sample or for a particular variable (e.g. tick species, collection locality, 
life stage, host). The most abundant bacterial species that have been identified frequently in ticks include endosymbionts such as Coxiella spp. [13, 40, 51], Rickettsia spp. [42, 44, 51-54], Francisella spp. [42, 53] and "Candidatus Midichloria mitochondrii" [50]. However, pathogenic, environmental and skin-associated bacteria have also been reported as highly abundant [13, 44]. The number of reads obtained for each bacterial species are not necessarily representative of the number of microorganisms that were present in the tick due to the multicopy nature of $16 \mathrm{~S}$ [55]. Estimates of the number of bacterial species initially present could be made by correcting for the number of $16 \mathrm{~S}$ copies in each species, or by targeting a single copy gene (e.g. rpoB gene). However, PCR amplification bias occurs when the amplification efficiency of templates differ, which leads to over- or under-representation of template DNA sequences [56, 57], and this can impact diversity measures of the dataset [38]. PCR conditions can be optimised to reduce, but not eliminate, the levels of PCR bias, such as reducing temperature ramping speeds, extending the denaturation step to provide sufficient time for GC-rich DNA fragments to denature, adding betaine to keep GC-rich templates single-stranded and using different enzymes such as AccuPrime Taq HiFi (Thermo Fisher) to improve priming specificity [58]. Alternative nucleic acid amplification methods exist, but due to the lack of commercial availability [59], PCR is the mainstay of amplicon NGS library preparation procedures.

\section{The bacterial microbiome of ticks Bacterial pathogens}

Some of the most important bacterial TBPs include species of Anaplasma, Borrelia, Ehrlichia, Francisella and Rickettsia. Ixodes ricinus, the castor bean (or deer or sheep) tick, is prevalent throughout Europe and is responsible for transmitting a range of bacterial pathogens. Due to its significant role as a bacterial vector, I. ricinus has been commonly investigated using $16 S$ NGS [22, 23, 44, 50]. Ixodes ricinus ticks sampled from Lyme disease endemic regions were found to carry pathogenic species within the Borrelia burgdorferi (sensu lato) (s.l.) genogroup, including B. burgdorferi (sensu stricto) (s.s.), Borrelia garinii and Borrelia afzelii $[23,44,50]$; as well as Anaplasma phagocytophilum, Rickettsia helvetica and "Candidatus Neoehrlichia mikurensis" [23, 60]. Known TBPs are usually identified at species-specific loci with sensitive GPCR or multiplex PCR screening procedures that are faster and more affordable compared to NGS in diagnostic settings, i.e. when a tick has been removed from a patient with clinical illness [61]. Since 16S NGS can be harnessed as a broad screening tool for bacteria, it is a useful technique for non-hypothesis driven surveillance of bacterial TBPs. Although greater sensitivity with qPCR compared to NGS has been demonstrated in some studies $[62,63]$, other studies have shown that increasing the sequencing depth can increase the chances of detecting rare species [64].

Sequencing of universal genes with NGS also allows for novel species identification [50, 65]. Determining the pathogenicity of newly discovered bacteria is then challenging as the direct association between a particular clinical illness and the bacterial species, once within the vertebrate host, must be proven. To a limited extent, the potential for pathogenicity of novel species can be estimated at the molecular level, e.g. by how they cluster genetically with known TBPs and by the presence of 'pathogenicity islands' that code for virulence genes [66, 67]. Paradoxically, however, pathogenicity islands have also been found in non-pathogenic species [68]. The identification of novel microorganisms in ticks using NGS may lead in time to an increase in what has been termed 'reversed discovery of disease', whereby the microorganism is identified in ticks before its pathogenicity to animals or humans is established [69].

\section{Endosymbiotic bacteria}

Endosymbiotic bacteria that reside in hard ticks (ixodids) include species of Coxiella, Francisella, Rickettsia and "Candidatus Midichloria mitochondrii" [70]. Coxiellalike endosymbionts (CLEs) have been found in a variety of ixodids using NGS, including Amblyomma americanum (lone star tick) [71], Haemaphysalis flava [40], Ixodes ovatus [40], Rhipicephalus (Boophilus) microplus (cattle tick) [13], Rhipicephalus turanicus [51] and Rhipicephalus sanguineus (sensu lato) (s.l.) (brown dog tick) [51], and have also been found in soft tick (argasid) species, e.g. Ornithodoros spp. [60, 72]. Andreotti et al. [13] found that a CLE was most abundant in female $R$. microplus ovaries. Transovarial transmission of this CLE was recently demonstrated in Ornithodoros maritimus, A. americanum, $R$. sanguineus (s.l.) and $R$. microplus [72]. Although these CLEs purportedly have a symbiotic relationship with their tick hosts, a recent study by Angelakis et al. [73] detected a CLE from Rhipicephalus spp. ("Candidatus Coxiella massiliensis") in skin biopsies from people with clinical signs of fever, skin eschar and local lymph node enlargement, and this organism is now therefore linked with human infection [73]. The causative agent of $\mathrm{Q}$ fever in humans, Coxiella burnetii, is a relatively recent descendant of CLEs of ticks [72], and seven tick species have been experimentally demonstrated as competent vectors of C. burnetii [74].

Endosymbionts are an important component of the microbiome that can benefit tick survival. Rickettsial endosymbionts of Dermacentor andersoni (Rocky Mountain wood tick) and A. americanum, and the CLE of $A$. americanum are examples of endosymbionts that are 
essential for the survival and reproductive fitness of their host. Studies that exposed D. andersoni and A. americanum to antibiotics found that the exposed colony's progeny had lower numbers of their respective endosymbionts, which reduced the survival, feeding and moulting competence in $D$. andersoni [75], and reduced fecundity in $A$. americanum $[75,76]$. Although a complete understanding of the mechanisms that cause endosymbionts to promote tick survival and fecundity is lacking, endosymbionts may play a key role in providing essential nutrients and cofactors that are absent from the blood meal. For example, Smith et al. [77] sequenced the genome of the CLE of A. americanum to investigate the potential for nutrient-provisioning, and found that the genome encodes for major vitamin and cofactor biosynthesis pathways [77].

\section{Environmental and skin-associated bacteria}

It remains uncertain whether ubiquitous bacteria associated with soil, plants and skin that are frequently reported in NGS studies of ticks are contaminants from environmental or host sources, or whether they are genuinely associated with the tick microbiome. The tick's exoskeleton can be sterilised with bleach prior to nucleic acid extraction in an attempt to remove contaminant DNA, or bioinformatics pipelines can be used to remove contaminating reads that are present in extraction and no-template controls [23]. Studies that have used sterilisation techniques (e.g. washing the ticks with $10 \%$ sodium hypochlorite) have, however, still detected environmental and skin bacteria in ticks $[44,50,78]$. This may be due to inadequate sterilisation (i.e. bacteria may remain hidden in crevices that are not exposed to the bleach solution during washing), or these bacteria may be ingested by ticks during feeding, therefore may be present in the tick midgut. As ticks spend the majority of their lives in the environment, saprophytic bacteria may be acquired at some point during their life cycle.

Bacterial contamination is a widespread issue among NGS datasets due to the sensitivity of this technology [79]. Stringent laboratory procedures must be followed during sample handling to minimise laboratory-derived contamination; the use of sterile gloves, workstations, laminar flow hoods, PCR grade water and pipette tips with filters are some examples of laboratory practices that can reduce contamination. Library preparation reagents can also be a source of contamination, e.g. ligases, polymerases and nucleotides are purified from bacteria, and Bradyrhizobium spp. have been reported in ultrapure water systems [79]. To ensure that these contaminants are controlled for, extraction and no-template controls should be included so that the contaminant sequences can be identified and removed bioinformatically from the dataset.

\section{Organ-associated bacteria}

While most 16S NGS studies of ticks have extracted DNA from entirely homogenised ticks (Table 1), some researchers have examined bacterial profiles of tick organs [13, 40, 53, 80, 81]. Microbial communities differ between anatomical regions within the tick, such as the midgut, reproductive tract and the salivary glands [13, 17, 53, 75, 80-83]. Most TBPs are transmitted to the vertebrate host via salivary secretions during blood feeding. An assessment with 16S NGS of the bacteria present in the salivary glands of $I$. ovatus, Ixodes persulcatus (Taiga tick) and $H$. flava collected in Japan revealed a surprisingly large number of bacterial genera: 71 (I. ovatus), 127 (I. persulcatus) and 59 (H. flava) [40], and some of the medically important genera that were detected included Coxiella, Ehrlichia and Rickettsia [40].

Rickettsia parkeri, one of the causes of human rickettsiosis [84], is transmitted by Amblyomma maculatum (Gulf Coast tick), and has been detected in the midguts, salivary glands and saliva of questing ticks with NGS [53]. The study suggested that the bacteria may migrate from the midgut to the salivary glands prior to feeding [53], which differs from the transmission route of other pathogens, such as B. burgdorferi, which develops in the tick midgut of Ixodes spp. then translocates to the salivary glands during feeding $[85,86]$.

It is possible that dissected tick organs may be contaminated with microbes from surrounding tissues or adjacent organs. Methodologies to control for this should be implemented, otherwise alternative assays may need to be utilised e.g. fluorescence in situ hybridization (FISH) [87]. Immunofluorescence assays have provided insights into the transstadial transmission of a novel relapsing fever Borrelia sp. found in Amblyomma geoemydae in Japan, which was visualised in the midgut and salivary glands of instars after moulting [88].

\section{Neglected facets of the tick microbiome Viruses}

Viral species transmitted by ticks include tick-borne encephalitis virus [89], CCHFV [90], KFDV [91], Alkhurma virus, severe fever with thrombocytopenia syndrome virus (SFTSV) [92], Heartland virus (HRTV) [93], Powassan and Colorado tick fever viruses [94, 95], Africa swine fever virus, Nairobi sheep disease virus (NSDV) and Louping ill virus $[5,6]$. Shotgun sequencing of viral cDNA is a more comprehensive approach than amplicon sequencing for studying the tick virome due to the lack of universal genes in viruses [96]. Thus far, only four shotgun NGS studies have investigated the virome of ticks [97-100]. Xia et al. [97] sequenced the virome of Rhipicephalus spp. using the Ion Torrent platform in China and discovered novel anellovirus and nairovirus species. The most abundant sequences were nairoviruses, 
phages and invertebrate viruses [97]. Most of the recognised nairoviruses are transmitted by ticks, and some that impact human and animal health, such as CCHFV and NSDV, have been identified in Rhipicephalus spp. previously [101]. Tokarz et al. [100] discovered eight previously uncharacterised viral sequences in I. scapularis, D. variabilis and $A$. americanum from the USA that were most similar to nairovirus, phlebovirus, invertebrate-like virus, mononegavirus and tetravirus-like virus, and also identified Powassan virus in I. scapularis [100]. Li et al. [98] and Shi et al. [99] investigated the viromes of a wide variety of invertebrates, including ticks from China, and their results suggest that arthropods are major reservoir hosts for negative-sense RNA viruses that are found in vertebrates, plants, fungi and protists. The considerable number of known tick-borne viruses and the growing number of discoveries of novel viruses potentially transmitted by ticks warrants a wider investigation of tick viromes.

\section{Eukaryotes}

Protozoan tick-borne diseases (TBDs), such as babesiosis and theileriosis, are caused by the piroplasms Babesia spp. and Theileria spp., respectively [102]. To date, no $18 \mathrm{~S}$ ribosomal RNA gene (18S) NGS studies on ticks have been published, and there have been no protozoan discoveries reported in shotgun sequencing studies on ticks. Shotgun sequencing could be used to identify protists in addition to viruses and bacteria; however, the relative abundance of eukaryotic tick DNA is much greater than protist DNA, and so a great sequencing depth would be required for protist detection. 18S ranges from $1.5 \mathrm{kbp}$ to more than $4.5 \mathrm{kbp}$ [103], and like bacterial 16S, $18 \mathrm{~S}$ contains nine hypervariable regions (V1-V9). The use of universal $18 \mathrm{~S}$ primers for identifying eukaryotic DNA with amplicon sequencing in ticks is challenging as other eukaryotic species from soil flora contaminants, animals and the tick itself will also be amplified. It may be possible to address this issue with blocking primers [50], or the use of protist-specific primers for NGS [104]. In addition to Babesia and Theileria, other Eukarya that require further characterisation at the community level in ticks include other apicomplexan species (e.g. Hepatozoon spp.), trypanosomes [105], fungi [106] and helminths [107].

\section{Factors influencing the microbiome}

Several studies have assessed the effect of environmental and host-related factors on tick microbiome diversity and composition. Environmental factors such as geographical location [41, 44-47], temperature, humidity [71], season [51], habitat type and soil type have been shown to influence the bacterial diversity and composition of ticks. Microbiome diversity and composition can also vary depending on vertebrate host and arthropod- related factors such as tick species [40-43], instar and sex [41, 44-47, 51, 54, 71, 81], anatomical location (e.g. midguts and salivary glands) $[13,53,75,81]$ and bloodfeeding $[52,108,109]$.

Other studies have assessed additional factors, such as the role of proteins in the microbiome that are encoded by the tick's genome, and their effect on pathogen acquisition. Silencing of transcripts using RNA interference (RNAi) of the antioxidant selenoproteins thioredoxin reductase (TrxR) and glutathione peroxidase (GPx) reduced the bacterial load of Rickettsiaceae in $A$. maculatum midguts and salivary glands [82], and $B$. burgdorferi in I. scapularis saliva [110], respectively. RNAi-mediated knockdown of the selenocysteinespecific elongation factor (SEF) gene in A. maculatum, which is important for selenoprotein translation, resulted in no detectable $R$. parkeri in A. maculatum midguts [111]. Other protein-encoding genes also affect pathogen acquisition and development, and are differentially regulated in ticks in response to Anaplasma marginale infection [112].

NGS investigations of ticks have also revealed that microbe-microbe interactions can influence microbiome composition. For example, Gurfield et al. [45] found that there was an inverse relationship between the number of Francisella-like endosymbionts (FLEs) and SFGR in Dermacentor occidentalis, which suggests that FLEs have an ability to interfere with SFGR colonisation [45]. Other non-NGS studies have demonstrated that endosymbionts can interfere with TBP transmission by affecting pathogen acquisition and colonisation [70, 113, 114]. Narasimhan et al. [83] altered the gut microbiota of $I$. scapularis by rearing larvae in sterile containers, and $16 \mathrm{~S} 454$ sequencing showed that the dysbiosed larvae had a higher abundance of Proteobacteria, including the genera Rickettsia, Thioclava and Delftia, compared to control larvae reared under normal conditions. In that study, qPCR assessments of larvae fed on B. burgdorferiinfected mice and pathogen-free mice showed that $B$. burgdorferi colonisation in dysbiosed larvae, as well as gentamycin-exposed larvae, was significantly reduced compared to the control larvae [83].

Similarly, a study by Gall et al. [17] showed that higher proportions of the endosymbiont Rickettsia bellii in D. andersoni were correlated with decreased acquisition of the pathogen $A$. marginale. However, generalisations cannot be made about the effect of all types of endosymbionts on pathogen acquisition across various tick species. Conversely, it was shown that the pathogen acquisition of Francisella novicida was positively correlated with high proportions of FLEs in D. andersoni [17].

Pathogens can also influence the tick gut and its microbiota. In I. scapularis, the pathogen A. phagocytophilum induces the protein I. scapularis antifreeze 
glycoprotein (IAFGP) [115], and was shown in a study by Abraham et al. [108] to decrease the abundance of the genera Enterococcus and Rickettsia and increase Pseudomonas in I. scapularis midguts. The study by Abraham et al. [108] also showed that A. phagocytophilum decreased the expression levels of peritrophin-1, peritrophin- 2 and peritrophin-4, which are genes that encode for peritrophin, a major component of peritrophic matrix (PM) glycoproteins that form a layer separating epithelial cells from the tick gut lumen, and this caused a decrease in the thickness of the PM. This study demonstrated that $A$. phagocytophilum induced changes in the gut barrier by decreasing peritrophin expression, which enhanced the colonisation of $A$. phagocytophilum in the tick midgut [108].

Macroparasites of ticks may also influence the microbiome. It has been suggested that Wolbachia pipientis may not be a naturally occurring endosymbiont of ticks, but rather has been introduced to the tick microbiome by a parasitic wasp [116]. Further studies are required to establish the extent to which environmental, tick and vertebrate host-related factors influence the microbiome. Other variables that could also be considered include degree of engorgement, gut protein expression responses to a blood meal $[117,118]$, host immune molecules present in blood meals [119], tick gut changes in response to feeding [120], tick copulation and egg fertilisation $[120,121]$, vertebrate host skin microflora, microorganisms in the vertebrate host's blood and community changes brought about by microbial interactions within the tick.

\section{Implications of microbiome studies}

Not only is NGS a comprehensive tool for studying tick microbiomes, it can also be used for TBP or 'pathobiome' surveillance to improve TBD diagnostics [122] and for the reversed discovery of human and animal TBDs. Importantly, an understanding of the factors that influence the microbiome, and the role of the microbiome, provides new avenues to be explored for TBD control. Most tick and TBD control strategies focus on the use of acaricides and vaccines [123-125]; however, tick populations can become resistant to chemical acaricides [123], and vaccine development takes an average of ten years [126]. Strategies could be developed to manipulate the tick microbiome to decrease the vectorial capacity of ticks by hindering pathogen acquisition, development, and horizontal and vertical transmission, which could have a long-term impact on TBP transmission and could ultimately reduce morbidity and mortality caused by TBDs. In the field of human microbiome research, investigations of metabolic, signaling and immune interactions between gut microbes and host physiology have led to the concept of therapeutic microbial manipulation to treat or prevent diseases [127-129]. For example, faecal microbiota transplantations have been successfully used to reestablish colonic microbial populations that fight against intestinal infections with Clostridium difficile [130].

Microbiome alterations have also been induced in arthropod vectors to combat vector-borne diseases. The endosymbiont $W$. pipientis causes cytoplasmic incompatibility and has been introduced into mosquito vectors such as Culex pipiens, Aedes aegypti, Aedes albopictus and Aedes polynesiensis to control their populations with the aim to reduce the transmission of diseases such as filariasis, dengue fever, yellow fever, chikungunya and Zika fever. There are ongoing trials and evaluations of releasing Wolbachia-infected Ae. aegypti in Brazil, Colombia, Indonesia, Singapore and Vietnam [131]. Similar microbial management strategies could be developed for ticks that promote the growth of endosymbiotic bacteria, such as $R$. bellii, to reduce acquisition of pathogens, such as $A$. marginale in $D$. andersoni, or strategies could be developed to the hinder the growth of FLEs in $D$. andersoni to reduce F. novidica acquisition [17]. Genes that could be manipulated to hinder pathogen acquisition and development include antioxidant selenoprotein genes and PM glycoprotein genes. Silencing of TrxR in A. maculatum and GPx in I. scapularis negatively affects $R$. parkeri and B. burgdorferi development, respectively $[82,110,111]$. Preventing $A$. phagocytophilum from decreasing the expression levels of peritrophin-1, peritrophin-2 and peritrophin4 would impede A. phagocytophilum growth in the tick midgut [108].

\section{Conclusions}

Next-generation sequencing methodology is a powerful technique that is revolutionising our understanding of TBPs, endosymbiotic bacteria and other microbes associated with the tick microbiome. Identification of species that form this arthropod's microbiome is fundamental to exploring its functions, yet despite this, there is currently a dearth of published studies investigating viruses and eukarya in ticks. Additionally, as this research is still in its infancy, the microbiomes of many tick species remain to be investigated. The influences of the environment, the vertebrate host and the tick itself on the microbial diversity and composition in ticks need to be further defined and assessed. This is an important consideration for study design and will have an impact on the interpretation and biological relevance of the findings. Further tick microbiome research is required to increase our understanding of the molecular and biochemical basis of tick microbiome interactions, and as this 
improves, novel microbial management strategies for TBDs may be developed in the future.

\section{Abbreviations}

16S: 165 ribosomal ribonucleic acid gene; 18S: 18S ribosomal ribonucleic acid gene; CCHFV: Crimean-Congo haemorrhagic fever virus; CDNA: complementary DNA; CLE: Coxiella-like endosymbiont;

FISH: fluorescence in situ hybridization; FLE: Francisella-like endosymbiont Gbp: gigabase pairs; gDNA: genomic deoxyribonucleic acid; GPx: glutathione peroxidase; HRTV: Heartland virus; IAFGP: Ixodes scapularis antifreeze glycoprotein; Kbp: kilobase pairs; KFDV: Kyasanur Forest disease virus; NGS: next-generation sequencing; NSDV: Nairobi sheep disease virus; OTU: operational taxonomic unit; PM: peritrophic matrix; QIIME: Quantitative Insights Into Microbial Ecology; qPCR: real-time polymerase chain reaction; RNAi: ribonucleic acid interference; SEF: selenocysteine-specific elongation factor; SFGR: spotted fever group rickettsiae; SFTSV: severe fever with thrombocytopenia syndrome virus; TBD: tick-borne disease; TBP: tick-borne pathogen; TrxR: thioredoxin reductase; V1-V9: hypervariable regions 1-9

\section{Acknowledgements}

We thank the Australian Research Council (Linkage Project 130100050), Bayer Australia Ltd., Bayer AG (Germany) and Murdoch University for supporting our tick microbiome research.

\section{Funding}

This research is funded by the Australian Research Council (Linkage Project $130,100,050)$, Bayer Australia Ltd. and Bayer AG (Germany). TLG is supported by a Murdoch University Postgraduate Research scholarship.

\section{Availability of data and materials}

Not applicable.

\section{Authors' contributions}

TLG developed and wrote the manuscript. AWG and AP provided critical reviews and suggestions for the content. UMR, CLO and PJI guided and assisted in writing the manuscript. All authors read and approved the final manuscript.

\section{Ethics approval and consent to participate}

Not applicable.

\section{Consent for publication}

Not applicable.

\section{Competing interests}

The authors declare that they have no competing interests.

\section{Publisher's Note}

Springer Nature remains neutral with regard to jurisdictional claims in published maps and institutional affiliations.

Received: 18 September 2017 Accepted: 21 November 2017

Published online: 04 January 2018

\section{References}

1. Whipps JM, Lewis K, Cooke RC. Mycoparasitism and plant disease control. In: Burge MN, editor. Fungi in biological control systems. Manchester: Manchester University Press. 1988:176.

2. Peterson J, Garges S, Giovanni M, Mclnnes P, Wang L, Schloss JA, et al. The NIH human microbiome project. Genome Res. 2009;19:2317-23.

3. Ursell LK, Metcalf JL, Parfrey LW, Knight R. Defining the human microbiome. Nutr Rev. 2012;70:38-44

4. Narasimhan S, Fikrig E. Tick microbiome: the force within. Trends Parasitol. 2015:31:315-23.

5. de la Fuente J, Estrada-Pena A, Venzal JM, Kocan KM, Sonenshine DE. Overview: ticks as vectors of pathogens that cause disease in humans and animals. Front Biosci. 2008;13:6938-46.

6. Dantas-Torres F, Chomel BB, Otranto D. Ticks and tick-borne diseases: a one health perspective. Trends Parasitol. 2012;28:437-46.
7. Brites-Neto J, Duarte KMR, Martins TF. Tick-borne infections in human and animal population worldwide. Vet World. 2015:8:301-15.

8. Noda H, Munderloh UG, Kurtti TJ. Endosymbionts of ticks and their relationship to Wolbachia spp. and tick-borne pathogens of humans and animals. Appl Environ Microbiol. 1997;63:3926-32.

9. Sacchi L, Bigliardi E, Corona S, Beninati T, Lo N, Franceschi A. A symbiont of the tick Ixodes ricinus invades and consumes mitochondria in a mode similar to that of the parasitic bacterium Bdellovibrio bacteriovorus. Tissue Cell. 2004;36:43-53.

10. Scoles GA. Phylogenetic analysis of the Francisella-like endosymbionts of Dermacentor ticks. J Med Entomol. 2004:41:277-86.

11. Berlec A. Novel techniques and findings in the study of plant microbiota: search for plant probiotics. Plant Sci. 2012;193-194:96-102.

12. Kim Y, Koh I, Rho M. Deciphering the human microbiome using nextgeneration sequencing data and bioinformatics approaches. Methods. 2015; 79-80:52-9.

13. Andreotti R, Pérez de León AA, Dowd SE, Guerrero FD, Bendele KG, Scoles GA. Assessment of bacterial diversity in the cattle tick Rhipicephalus (Boophilus) microplus through tag-encoded pyrosequencing. BMC Microbiol. 2011;11:6.

14. Roche Diagnostics Corporation. http://http://www.my454.com//. Accessed 15 May 2017

15. Illumina. http://www.illumina.com/. Accessed 15 May 2017.

16. Thermo Fisher Scientific. http://www.thermofisher.com/. Accessed 15 May 2017

17. Gall CA, Reif KE, Scoles GA, Mason KL, Mousel M, Noh SM, et al. The bacterial microbiome of Dermacentor andersoni ticks influences pathogen susceptibility. ISME J. 2016;

18. Goodwin S, McPherson JD, McCombie WR. Coming of age: ten years of next-generation sequencing technologies. Nat Rev Genet. 2016;17:333-51

19. Roumpeka DD, Wallace RJ, Escalettes F, Fotheringham I, Watson MA. Review of bioinformatics tools for bio-prospecting from metagenomic sequence data. Front Genet. 2017:8:23.

20. Pacific Biosciences of California. http://www.pacb.com/. Accessed 15 May 2017

21. Oxford Nanopore Technologies. https://nanoporetech.com/. Accessed 15 May 2017

22. Nakao R, Abe T, Nijhof AM, Yamamoto S, Jongejan F, Ikemura T, et al. A novel approach, based on BLSOMs (batch learning self-organizing maps), to the microbiome analysis of ticks. ISME J. 2013;7:1003-15.

23. Vayssier-Taussat M, Moutailler S, Michelet L, Devillers E, Bonnet S, Cheval J, et al. Next generation sequencing uncovers unexpected bacterial pathogens in ticks in Western Europe. PLoS One. 2013;8:e81439.

24. Jones MB, Highlander SK, Anderson EL, Li W, Dayrit M, Klitgord N, et al. Library preparation methodology can influence genomic and functional predictions in human microbiome research. Proc Natl Acad Sci USA. 2015; 112:14024-9.

25. Lim YW, Haynes M, Furlan M, Robertson CE, Harris JK, Rohwer F. Purifying the impure: sequencing metagenomes and metatranscriptomes from complex animal-associated samples. J Vis Exp. 2014:52117.

26. Carpi G, Walter KS, Bent SJ, Hoen AG, Diuk-Wasser M, Caccone A. Whole genome capture of vector-borne pathogens from mixed DNA samples: a case study of Borrelia burgdorferi. BMC Genomics. 2015;16:434

27. Moen AEF, Tannæs TM, Vatn S, Ricanek P, Vatn MH, Jahnsen J. Simultaneous purification of DNA and RNA from microbiota in a single colonic mucosal biopsy. BMC Res Notes. 2016;9:328.

28. Mertes F, ElSharawy A, Sauer S, van Helvoort JM, van der Zaag PJ, Franke A, et al. Targeted enrichment of genomic DNA regions for next-generation sequencing. Brief Funct Genomics. 2011;10:374-86.

29. Siegwald L, Touzet $H$, Lemoine $Y$, Hot D, Audebert C, Caboche S. Assessment of common and emerging bioinformatics pipelines for targeted metagenomics. PLoS One. 2017:12:e0169563.

30. Whittaker RH. Evolution and measurement of species diversity. Taxon. 1972;21:213-51.

31. Lozupone C, Knight R. UniFrac: a new phylogenetic method for comparing microbial communities. Appl Environ Microbiol. 2005;71:8228-35.

32. Lozupone CA, Hamady M, Kelley ST, Knight R. Quantitative and qualitative beta diversity measures lead to different insights into factors that structure microbial communities. Appl Environ Microbiol. 2007;73:1576-85.

33. Claesson MJ, Wang Q, O'Sullivan O, Greene-Diniz R, Cole JR, Ross RP, et al. Comparison of two next-generation sequencing technologies for resolving 
highly complex microbiota composition using tandem variable 16S rRNA gene regions. Nucleic Acids Res. 2010;38:e200-e.

34. Clarridge JE. Impact of $16 \mathrm{~S}$ rRNA gene sequence analysis for identification of bacteria on clinical microbiology and infectious diseases. Clin Microbiol Rev. 2004;17:840-62.

35. Chakravorty S, Helb D, Burday M, Connell N, Alland DA. Detailed analysis of $16 \mathrm{~S}$ ribosomal RNA gene segments for the diagnosis of pathogenic bacteria. J Microbiol Methods. 2007:69:330-9.

36. Sperling JL, Silva-Brandão KL, Brandão MM, Lloyd VK, Dang S, Davis CS, et al. Comparison of bacterial 165 rRNA variable regions for microbiome surveys of ticks. Ticks Tick Borne Dis. 2017;8:453-61.

37. Raoult D, Roux V. Rickettsioses as paradigms of new or emerging infectious diseases. Clin Microbiol Rev. 1997;10:694-719.

38. Sun $Y$, Cai $Y$, Huse SM, Knight $R$, Farmerie WG, Wang $X$, et al. A large-scale benchmark study of existing algorithms for taxonomy-independent microbial community analysis. Brief Bioinform. 2012;13:107-21.

39. Schmidt TSB, Matias Rodrigues JF, von Mering C. Ecological consistency of SSU rRNA-based operational taxonomic units at a global scale. PLoS Comput Biol. 2014;10:e1003594.

40. Qiu Y, Nakao R, Ohnuma A, Kawamori F, Sugimoto C. Microbial population analysis of the salivary glands of ticks; a possible strategy for the surveillance of bacterial pathogens. PLoS One. 2014;9:e103961.

41. Van Treuren W, Ponnusamy L, Brinkerhoff RJ, Gonzalez A, Parobek CM Juliano JJ, et al. Variation in the microbiota of Ixodes ticks with regard to geography, species, and sex. Appl Environ Microbiol. 2015;81:6200-9.

42. Hawlena H, Rynkiewicz E, Toh E, Alfred A, Durden LA, Hastriter MW, et al. The arthropod, but not the vertebrate host or its environment, dictates bacterial community composition of fleas and ticks. ISME J. 2013;7:221-3.

43. Rynkiewicz EC, Hemmerich C, Rusch DB, Fuqua C, Clay K. Concordance of bacterial communities of two tick species and blood of their shared rodent host. Mol Ecol. 2015;24:2566-79.

44. Carpi G, Cagnacci F, Wittekindt NE, Zhao F, Qi J, Tomsho LP, et al. Metagenomic profile of the bacterial communities associated with Ixodes ricinus ticks. PLoS One. 2011;6:e25604.

45. Gurfield N, Grewal S, Cua LS, Torres PJ, Kelley ST. Endosymbiont interference and microbial diversity of the Pacific coast tick, Dermacentor occidentalis, in San Diego County, California. PeerJ. 2017;5:e3202.

46. Williams-Newkirk AJ, Rowe LA, Mixson-Hayden TR, Dasch GA. Characterization of the bacterial communities of life stages of free living lone star ticks (Amblyomma americanum). PLoS One. 2014;9:e102130

47. Trout Fryxell RT, DeBruyn JM. The microbiome of Ehrlichia infected and uninfected lone star ticks (Amblyomma americanum). PLoS One. 2016;11:e0146651.

48. Khoo JJ, Chen F, Kho KL, Ahmad Shanizza Al, Lim FS, Tan KK, et al. Bacterial community in Haemaphysalis ticks of domesticated animals from the orang Asli communities in Malaysia. Ticks Tick Borne Dis. 2016;7:929-37.

49. Vestheim $H$, Jarman SN. Blocking primers to enhance PCR amplification of rare sequences in mixed samples - a case study on prey DNA in Antarctic krill stomachs. Front Zool. 2008;5:12.

50. Gofton AW, Oskam CL, Lo N, Beninati T, Wei H, McCarl V, et al. Inhibition of the endosymbiont "Candidatus Midichloria mitochondrii" during 16S rRNA gene profiling reveals potential pathogens in Ixodes ticks from Australia. Parasit Vectors. 2015;8:345.

51. Lalzar I, Harrus S, Mumcuoglu KY, Gottlieb Y. Composition and seasonal variation of Rhipicephalus turanicus and Rhipicephalus sanguineus bacterial communities. Appl Environ Microbiol. 2012;78:4110-6.

52. Zhang X-C, Yang Z-N, Lu B, Ma X-F, Zhang C-X, H-J X. The composition and transmission of microbiome in hard tick, lxodes persulcatus, during blood meal. Ticks Tick Borne Dis. 2014:5:864-70.

53. Budachetri K, Browning RE, Adamson SW, Dowd SE, Chao C-C, Ching W-M, et al. An insight into the microbiome of the Amblyomma maculatum (Acari: Ixodidae). J Med Entomol. 2014;51:119-29.

54. Ponnusamy L, Gonzalez A, Van Treuren W, Weiss S, Parobek CM, Juliano $J$ J, et al. Diversity of rickettsiales in the microbiome of the lone star tick, Amblyomma americanum. Appl Environ Microbiol. 2014;80:354-9.

55. Kembel SW, Wu M, Eisen JA, Green JL. Incorporating 165 gene copy number information improves estimates of microbial diversity and abundance. PLoS Comput Biol. 2012;8:e1002743.

56. Hong S, Bunge J, Leslin C, Jeon S, Epstein SS. Polymerase chain reaction primers miss half of rRNA microbial diversity. ISME J. 2009;3:1365-73.
57. Nadkarni MA, Martin FE, Hunter N, Jacques NA. Methods for optimizing DNA extraction before quantifying oral bacterial numbers by real-time PCR. FEMS Microbiol Lett. 2009;296:45-51.

58. Aird D, Ross MG, Chen W-S, Danielsson M, Fennell T, Russ C, et al. Analyzing and minimizing PCR amplification bias in Illumina sequencing libraries. Genome Biol. 2011;12:R18-R.

59. Fakruddin M, Mannan KSB, Chowdhury A, Mazumdar RM, Hossain MN, Islam $S$, et al. Nucleic acid amplification: alternative methods of polymerase chain reaction. J Pharm Bioallied Sci. 2013;5:245-52.

60. Almeida AP, Marcili A, Leite RC, Nieri-Bastos FA, Domingues LN, Martins JR, et al. Coxiella symbiont in the tick Ornithodoros rostratus (Acari: Argasidae). Ticks Tick Borne Dis. 2012;3:203-6.

61. Mediannikov O, Fenollar F. Looking in ticks for human bacterial pathogens. Microb Pathog. 2014;77:142-8.

62. Lecuit $\mathrm{M}$, Eloit $\mathrm{M}$. The diagnosis of infectious diseases by whole genome next generation sequencing: a new era is opening. Front Cell Infect Microbiol. 2014:4:25.

63. Wylie KM, Mihindukulasuriya KA, Sodergren E, Weinstock GM, Storch GA. Sequence analysis of the human virome in febrile and afebrile children. PLoS One. 2012;7:e27735.

64. Salipante SJ, Sengupta DJ, Rosenthal C, Costa G, Spangler J, Sims EH, et al. Rapid 16S rRNA next-generation sequencing of polymicrobial clinical samples for diagnosis of complex bacterial infections. PLoS One. 2013;8:e65226

65. Gofton AW, Doggett S, Ratchford A, Oskam CL, Paparini A, Ryan U, et al. Bacterial profiling reveals novel "Ca. Neoehrlichia", Ehrlichia, and Anaplasma species in Australian human-biting ticks. PLoS One. 2015;10:e0145449.

66. Knapp S, Hacker J, Jarchau T, Goebel W. Large, unstable inserts in the chromosome affect virulence properties of uropathogenic Escherichia coli O6 strain 536. J Bacteriol. 1986;168:22-30.

67. Hacker J, Bender L, Ott M, Wingender J, Lund B, Marre R, et al. Deletions of chromosomal regions coding for fimbriae and hemolysins occur in vitro and in vivo in various extraintestinal Escherichia coli isolates. Microb Pathog. 1990;8:213-25.

68. Gennari M, Ghidini V, Caburlotto G, Lleo MM. Virulence genes and pathogenicity islands in environmental Vibrio strains nonpathogenic to humans. FEMS Microbiol Ecol. 2012:82:563-73.

69. Tijsse-Klasen E, Koopmans MPG, Sprong H. Tick-borne pathogen - reversed and conventional discovery of disease. Front Public Health. 2014;2:73.

70. Ahantarig A, Trinachartvanit W, Baimai V, Grubhoffer L. Hard ticks and their bacterial endosymbionts (or would be pathogens). Folia Microbiol. 2013:58:419-28.

71. Menchaca AC, Visi DK, Strey OF, Teel PD, Kalinowski K, Allen MS, et al. Preliminary assessment of microbiome changes following blood-feeding and survivorship in the Amblyomma americanum nymph-to-adult transition using semiconductor sequencing. PLoS One. 2013;8:e67129.

72. Duron O, Noel V, McCoy KD, Bonazzi M, Sidi-Boumedine K, Morel O, et al. The recent evolution of a maternally-inherited endosymbiont of ticks led to the emergence of the $\mathrm{Q}$ fever pathogen, Coxiella burnetii. PLoS Pathog. 2015;11:e1004892.

73. Angelakis E, Mediannikov O, Jos S-L, Berenger J-M, Parola P, Raoult D. Candidatus Coxiella massiliensis infection. Emerging. Infect Dis. 2016;22:285.

74. Duron O, Sidi-Boumedine K, Rousset E, Moutailler S, Jourdain E. The importance of ticks in $\mathrm{Q}$ fever transmission: what has (and has not) been demonstrated? Trends Parasitol. 2015:31:536-52.

75. Clayton KA, Gall CA, Mason KL, Scoles GA, Brayton KA. The characterization and manipulation of the bacterial microbiome of the Rocky Mountain wood tick, Dermacentor andersoni. Parasit Vectors. 2015;8:632.

76. Zhong J, Jasinskas A, Barbour AG. Antibiotic treatment of the tick vector Amblyomma americanum reduced reproductive fitness. PLoS One. 2007;2:e405.

77. Smith TA, Driscoll T, Gillespie JJ, Raghavan RA. Coxiella-like endosymbiont is a potential vitamin source for the lone star tick. Genome Biol Evol. 2015:7:831-8.

78. Heise SR, Elshahed MS, Little SE. Bacterial diversity in Amblyomma americanum (Acari: Ixodidae) with a focus on members of the genus Rickettsia. J Med Entomol. 2010;47:258-68.

79. Strong MJ, Xu G, Morici L, Splinter Bon-Durant S, Baddoo M, Lin Z, et al. Microbial contamination in next generation sequencing: implications for sequence-based analysis of clinical samples. PLoS Path. 2014:10:e1004437.

80. Budachetri K, Gaillard D, Williams J, Mukherjee N, Karim SA. Snapshot of the microbiome of Amblyomma tuberculatum ticks infesting the gopher tortoise, an endangered species. Ticks Tick Borne Dis. 2016;7:1225-9. 
81. Zolnik CP, Prill RJ, Falco RC, Daniels TJ, Kolokotronis S-O. Microbiome changes through ontogeny of a tick pathogen vector. Mol Ecol. 2016;25:4963-77.

82. Budachetri K, Karim S. An insight into the functional role of thioredoxin reductase, a selenoprotein, in maintaining normal native microbiota in the Gulf coast tick (Amblyomma maculatum). Insect Mol Biol. 2015;24:570-81.

83. Narasimhan S, Rajeevan N, Liu L, Zhao Yang O, Heisig J, Pan J, et al. Gut microbiota of the tick vector Ixodes scapularis modulate colonization of the Lyme disease spirochete. Cell Host Microbe. 2014;15:58-71.

84. Paddock CD, Fournier P-E, Sumner JW, Goddard J, Elshenawy Y, Metcalfe MG, et al. Isolation of Rickettsia parkeri and identification of a novel spotted fever group Rickettsia sp. from Gulf Coast ticks (Amblyomma maculatum) in the United States. Appl Environ Microbiol. 2010;76:2689-96.

85. De Silva AM, Fikrig E. Growth and migration of Borrelia burgdorferi in Ixodes ticks during blood feeding. Am J Trop Med Hyg. 1995;53:397-404.

86. Gray JS. Review the ecology of ticks transmitting Lyme borreliosis. Exp Appl Acarol. 1998:22:249-58.

87. Klyachko O, Stein BD, Grindle N, Clay K, Fuqua C. Localization and visualization of a Coxiella-type symbiont within the lone star tick, Amblyomma americanum. Appl Environ Microbiol. 2007;73:6584-94.

88. Takano A, Sugimori C, Fujita H, Kadosaka T, Taylor KR, Tsubota T, et al. A novel relapsing fever Borrelia sp. infects the salivary glands of the molted hard tick, Amblyomma geoemydae. Ticks Tick Borne Dis. 2012;3:259-61.

89. Havlikova S, Lickova M, Klempa B. Non-viraemic transmission of tick-borne viruses. Acta Virol. 2013;57:123-9.

90. Bente DA, Forrester NL, Watts DM, McAuley AJ, Whitehouse CA, Bray M. Crimean-Congo hemorrhagic fever: history, epidemiology, pathogenesis, clinical syndrome and genetic diversity. Antivir Res. 2013;100:159-89.

91. Holbrook MR. Kyasanur Forest disease. Antivir Res. 2012;96:353-62.

92. XJ Y, Liang MF, Zhang SY, Liu Y, Li JD, Sun YL, et al. Fever with thrombocytopenia associated with a novel bunyavirus in China. N Engl J Med. 2011;364:1523-32.

93. McMullan LK, Folk SM, Kelly AJ, MacNeil A, Goldsmith CS, Metcalfe MG, et al. A new phlebovirus associated with severe febrile illness in Missouri. N Engl Med. 2012;367:834-41.

94. Meagher KE, Decker CF. Other tick-borne illnesses: tularemia, Colorado tick fever, tick paralysis. Dis Mon. 2012;58:370-6.

95. Stromdahl EY, Hickling GJ. Beyond Lyme: aetiology of tick-borne human diseases with emphasis on the south-eastern united states. Zoonoses Public Health. 2012;59:48-64.

96. Uyaguari-Diaz MI, Chan M, Chaban BL, Croxen MA, Finke JF, Hill JE, et al. A comprehensive method for amplicon-based and metagenomic characterization of viruses, bacteria, and eukaryotes in freshwater samples. Microbiome. 2016;4:20.

97. Xia H, Hu C, Zhang D, Tang S, Zhang Z, Kou Z, et al. Metagenomic profile of the viral communities in Rhipicephalus spp. ticks from Yunnan, China. PLoS One. 2015;10:e0121609.

98. Li C-X, Shi M, Tian J-H, Lin X-D, Kang Y-J, Chen L-J, et al. Unprecedented genomic diversity of RNA viruses in arthropods reveals the ancestry of negative-sense RNA viruses. elife. 2015;4:e05378.

99. Shi M, Lin X-D, Tian J-H, Chen L-J, Chen X, Li C-X, et al. Redefining the invertebrate RNA virosphere. Nature. 2016; doi:https://doi.org/10.1038/ nature20167.

100. Tokarz R, Williams SH, Sameroff S, Sanchez Leon M, Jain K, Lipkin WI. Virome analysis of Amblyomma americanum, Dermacentor variabilis, and Ixodes scapularis ticks reveals novel highly divergent vertebrate and invertebrate viruses. J Virol. 2014;88:11480-92.

101. Labuda M, Nuttall PA. Tick-borne viruses. Parasitology. 2004;129(Suppl 1): S221-45.

102. Jongejan F, Uilenberg G. The global importance of ticks. Parasitology. 2004;129(Suppl 1):S3-14.

103. Xie Q, Lin J, Qin Y, Zhou J, Structural BW. Diversity of eukaryotic 18S rRNA and its impact on alignment and phylogenetic reconstruction. Protein Cell. 2011:2:161-70

104. Ishaq SL. Wright A-DG. Design and validation of four new primers for nextgeneration sequencing to target the $18 \mathrm{~S}$ rRNA genes of gastrointestinal ciliate protozoa. Appl Environ Microbiol. 2014;80:5515-21.

105. Austen JM, Ryan UM, Friend JA, Ditcham WG, Reid SA. Vector of Trypanosoma copemani identified as /xodes sp. Parasitology. 2011;138:866-72.
106. Greengarten PJ, Tuininga AR, Morath SU, Falco RC, Norelus H, Daniels TJ. Occurrence of soil- and tick-borne fungi and related virulence tests for pathogenicity to Ixodes scapularis (Acari: Ixodidae). J Med Entomol. 2011:48:337-44.

107. Otranto D, Brianti E, Latrofa MS, Annoscia G, Weigl S, Lia RP, et al. On a Cercopithifilaria sp. transmitted by Rhipicephalus sanguineus: a neglected, but widespread filarioid of dogs. Parasit Vectors. 2012;5:1.

108. Abraham NM, Liu L, Jutras BL, Yadav AK, Narasimhan S, Gopalakrishnan V, et al. Pathogen-mediated manipulation of arthropod microbiota to promote infection. Proc Natl Acad Sci USA. 2017;114:E781-E90.

109. Swei A, Kwan JY. Tick microbiome and pathogen acquisition altered by host blood meal. ISME J. 2017;11:813-6.

110. Narasimhan S, Sukumaran B, Bozdogan U, Thomas V, Liang X, DePonte K, et al. A tick antioxidant facilitates the Lyme disease agent's successful migration from the mammalian host to the arthropod vector. Cell Host Microbe. 2007;2:7-18.

111. Adamson SW, Browning RE, Budachetri K, Ribeiro JM, Karim S. Knockdown of selenocysteine-specific elongation factor in Amblyomma maculatum alters the pathogen burden of rickettsia parkeri with epigenetic control by the Sin3 histone deacetylase corepressor complex. PLoS One. 2013;8:e82012.

112. de la Fuente J, Blouin EF, Manzano-Roman R, Naranjo V, Almazan C, Perez de la Lastra JM, et al. Functional genomic studies of tick cells in response to infection with the cattle pathogen, Anaplasma marginale. Genomics. 2007:90:712-22

113. Dib L, Bitam I, Tahri M, Bensouilah M, De Meeus T. Competitive exclusion between piroplasmosis and anaplasmosis agents within cattle. PLoS Pathog. 2008;4:e7.

114. Telford SR, Wormser GP. Bartonella spp. transmission by ticks not established. Emerg Infect Dis. 2010;16:379-84.

115. Heisig M, Abraham NM, Liu L, Neelakanta G, Mattessich S, Sultana H, et al. Antivirulence properties of an antifreeze protein. Cell Rep. 2014;9:417-24.

116. Plantard O. Detection of Wolbachia in the tick Ixodes ricinus is due to the presence of the hymenoptera endoparasitoid Ixodiphagus hookeri. PLoS One. 2014;7:e30692.

117. Anderson JM, Sonenshine DE, Valenzuela JG. Exploring the mialome of ticks: an annotated catalogue of midgut transcripts from the hard tick, Dermacentor variabilis (Acari: Ixodidae). BMC Genomics. 2008;9:552.

118. Hajdusek O, Sima R, Ayllon N, Jalovecka M, Perner J, de la Fuente J, et al. Interaction of the tick immune system with transmitted pathogens. Front Cell Infect Microbiol. 2013;3:26.

119. Grigor'eva LA. Morphofunctional changes in the midgut of tick females of the genus /xodes (Acari: Ixodidae) during and after feeding. Parazitologia. 2003;37:169-76.

120. Afzelius BA, Alberti G, Dallai R, Godula J, Witalinski W. Virus- and Rickettsiainfected sperm cells in arthropods. J Invertebr Pathol. 1989:53:365-77.

121. Otti O, Naylor RA, Siva-Jothy MT, Reinhardt K. Bacteriolytic activity in the ejaculate of an insect. Am Nat. 2009;174:292-5.

122. Vayssier-Taussat M, Kazimirova M, Hubalek Z, Hornok S, Farkas R, Cosson J-F, et al. Emerging horizons for tick-borne pathogens: from the 'one pathogenone disease' vision to the pathobiome paradigm. Future Microbiol. 2015;10:2033-43.

123. de la Fuente J, Contreras M. Tick vaccines: current status and future directions. Expert Rev Vaccines. 2015;14:1367-76.

124. Ghosh S, Azhahianambi P, Yadav MP. Upcoming and future strategies of tick control: a review. J Vector Borne Dis. 2007:44:79-89.

125. de la Fuente J, Kopáček P, Lew-Tabor A, Maritz-Olivier C. Strategies for new and improved vaccines against ticks and tick-borne diseases. Parasite Immunol. 2016;38:754-69.

126. Pronker ES, Weenen TC, Commandeur H, Claassen EHJHM, Osterhaus ADME Risk in vaccine Research and Development quantified. PLoS One. 2013;8:e57755.

127. Nicholson JK, Holmes E, Wilson ID. Gut microorganisms, mammalian metabolism and personalized health care. Nat Rev Micro. 2005;3:431-8.

128. Nicholson JK, Holmes E, Kinross J, Burcelin R, Gibson G, Jia W, et al. Host-gut microbiota metabolic interactions. Science. 2012;336:1262-7.

129. Boulangé $\mathrm{CL}$, Neves AL, Chilloux J, Nicholson JK, Dumas M-E. Impact of the gut microbiota on inflammation, obesity, and metabolic disease. Genome Med. 2016:8:42

130. Li YT, Cai HF, Wang ZH, Xu J, Fang JY. Systematic review with meta-analysis: long-term outcomes of faecal microbiota transplantation for Clostridium difficile infection. Aliment Pharmacol Ther. 2016;43:445-57. 
131. Huang Y-JS, Higgs S, Vanlandingham DL. Biological control strategies for mosquito vectors of arboviruses. Insects. 2017;8:21.

132. Tekin S, Dowd SE, Davinic M, Bursali A, Keskin A. Pyrosequencing based assessment of bacterial diversity in Turkish Rhipicephalus annulatus and Dermacentor marginatus ticks (Acari: Ixodidae). Parasitol Res. 2017;116:1055-61.

133. Budachetri K, Williams J, Mukherjee N, Sellers M, Moore F, Karim S. The microbiome of neotropical ticks parasitizing on passerine migratory birds. Ticks Tick Borne Dis. 2017;8:170-3.

Submit your next manuscript to BioMed Central and we will help you at every step:

- We accept pre-submission inquiries

- Our selector tool helps you to find the most relevant journal

- We provide round the clock customer support

- Convenient online submission

- Thorough peer review

- Inclusion in PubMed and all major indexing services

- Maximum visibility for your research

Submit your manuscript at www.biomedcentral.com/submit 